\title{
A propósito del formalismo de Johann von Neumann*
}

\author{
On the Formalism of Johann von Neumann
}

\author{
Abel Lassalle Casanave ${ }^{\dagger}$ \\ Luiz Carlos Pereira
}

\begin{abstract}
Resumen
En 1930, Johann von Neumann, junto con Rudolf Carnap y Arend Heyting, participó de un evento realizado en Königsberg llamado "Segundo Seminario sobre Epistemología de las Ciencias Exactas". La idea por detrás de la reunión de esos tres investigadores era presentar un cuadro suficientemente fiel de los tres programas de fundamentación de la matemática existentes al momento: el formalismo, el logicismo y el intuicionismo. El principal objetivo de este artículo es proponer un análisis del texto "La fundamentación formalista de la matemática" presentado por von Neumann en el mencionado seminario. Tratamos de mostrar como desde la perspectiva de von Neumann el problema de la consistencia de las teorías matemáticas es resultado de la transformación de una cuestión genuinamente filosófica en una genuina cuestión lógico-matemática y como una prueba de consistencia podía ser vista como la solución del problema fundacional. En la parte final del texto, presentamos algunas consideraciones acerca de las pruebas de consistencia de Gerhard Gentzen y su importancia para la teoría contemporánea de la demostración.
\end{abstract}

Palabras clave: formalismo - demostración de consistencia - Hilbert - von Neumann - Gentzen

\begin{abstract}
In 1930, Johann von Neumann, together with Rudolf Carnap and Arend Heyting, participated in a conference held in Königsberg, called "Second Seminar on the Epistemology of Exact Sciences". The idea behind the reunion of these three researchers was to compose a fairly faithful picture of the three main foundational programs of mathematics at the time: formalism, logicism, and intuitionism. The main objective of this paper is to propose an analysis of the text "The Formalist Foundation of Mathematics" presented by von Neumann in this conference. We show how, from von Neumann's perspective, the problem of the consistency of mathematical theories results from the transformation of a genuinely philosophical question into a genuinely logical-mathematical question and how a proof of consistency could be seen as the solution to the foundational problem. In the final part of the text, we present some considerations about Gerhard Gentzen's consistency proofs and their importance to contemporary proof theory.
\end{abstract}

Keywords: formalism - consistency proof - Hilbert - von Neumann - Gentzen

\footnotetext{
* Recibido: 23 de mayo de 2018. Aceptado con revisiones: 13 de septiembre de 2018.

† UFBA/CNPq, Brasil. Para contactar al autor, por favor, escribir a: abel.lassalle@gmail.com.

* PUC-Rio/UERJ/CNPq, Brasil. Para contactar al autor, por favor, escribir a: luiz@inf.puc-rio.br. Metatheoria 10(2)(2020): 51-59. ISSN 1853-2322. eISSN 1853-2330.

(C) Editorial de la Universidad Nacional de Tres de Febrero.

(C) Editorial de la Universidad Nacional de Quilmes.

Publicado en la República Argentina.
} 


\section{Introducción}

Que la llamada 'crisis de los fundamentos' decantó en la formulación de un problema matemático es algo que nunca debería olvidarse. Sin dejar de reconocer que la pregunta acerca de la confiabilidad (Zuverlässigkeit) de la matemática es una cuestión filosófica, von Neumann, al inicio de su contribución "La fundamentación formalista de la matemática", de 1931, así nos lo recuerda:

Resulta digno de atención que esta pregunta, en sí y por sí misma filosófica y epistemológica, se encuentra en condiciones de ser tratada como una pregunta lógico-matemática. (von Neumann 1931, p. $116)^{1}$

Von Neumann había sido convidado por los organizadores del Segundo Seminario sobre la Epistemología de las Ciencias Exactas, organizado por la Sociedad para la Filosofía Empirista, como representante de la posición formalista. El seminario, que tuvo lugar en Könnisberg del 5 al 7 de septiembre de 1930, contó también con la participación de Rudolf Carnap, representando la posición logicista, y Arend Heyting, representando la posición intuicionista. La contribución de las posiciones intuicionista (constructivista), logicista y formalista para que la cuestión de la confiabilidad de la matemática clásica se haya transformado en un problema matemático (o una serie de ellos) es recapitulada de la siguiente manera por von Neumann: en primer lugar, la crítica intuicionista de Brouwer a los métodos de la matemática clásica; en segundo, la descripción de Russell de los métodos de la misma por medio de un simbolismo, aunque sin distinguir entre ellos los buenos de los malos; finalmente, las investigaciones matemático-combinatorias de Hilbert sobre esos métodos y sus relaciones. La versión de von Neumann, esencialmente correcta, no deja de ser la del formalismo hilbertiano, que hasta ese momento al menos era el vencedor del debate. Las páginas que siguen analizan la contribución de von Neumann al mencionado seminario desde la perspectiva que se adquiere después de un siglo de fundamentos de la matemática.

\section{La cuestión de la confiabilidad de la matemática clásica}

Ahora bien, de hecho, si bien von Neumann lo formula en general en términos de la matemática clásica, el problema matemático específico a cuya solución en ese momento la escuela de Hilbert estaba abocada era el de la demostración de la consistencia de la aritmética de Peano, aunque ciertamente no hubiera dudas acerca de la confiabilidad de la misma. Habría entonces que observar que en este caso la demostración de la consistencia de la aritmética es el primer paso de una estrategia estratificada para la solución del problema general de la consistencia de la matemática clásica: aritmética, análisis, teoría de números transfinitos.

Ahora bien, ¿cómo confluyen el intuicionismo de Brouwer, el logicismo de Russell y el formalismo de Hilbert en la reducción de la pregunta filosófica acerca de la confiabilidad de la matemática clásica al problema matemático de la demostración de consistencia? Ciertamente, la crítica de Brouwer a los métodos clásicos, señaladamente a la validez irrestricta de tercero excluido, es un elemento relevante, aunque críticas a procedimientos que genéricamente son denominados infinitarios o transfinitos ya se encuentren, entre otros, en Kronecker y Poincaré. Hilbert había distinguido entre métodos finitarios confiables y métodos transfinitos acerca de los cuales su confiabilidad podría ser puesta en duda, pero en su artículo von Neumann simplemente identifica los primeros con los procedimientos intuicionísticamente aceptables. La cuestión, en principio, no es trivial, pues de lo que se trata es de indicar cuáles son los medios, qué herramientas demostrativas, son las aceptables para la solución (negativa o positiva, parcial o total) del problema. Para von Neumann se trata de demostrar la consistencia de la aritmética por métodos aceptables en la aritmética intuicionista o constructiva. Por

\footnotetext{
${ }^{1}$ En este volumen, p. 79.
} 
cierto, se podría argumentar que no hay ninguna razón para utilizar en la solución de un problema dado métodos deductivamente más débiles que aquellos que son aceptados por los adversarios.

El papel del logicismo de Russell es de naturaleza completamente diferente: en él, von Neumann, así como también Hilbert, encontraron un simbolismo adecuado para la formalización completa de teorías matemáticas, lógica clásica subyacente incluida. En particular, Hilbert introduce una noción de demostración en un sistema formal $F$ como secuencia de fórmulas de un lenguaje formal dado tal que cada una de ellas o bien es un axioma o bien se sigue de fórmulas anteriores en la secuencia por una regla de inferencia. Así, el sistema formal de que se trate y sus demostraciones se pueden tornar ahora ellos mismos objetos matemáticos. Y entonces el problema puede ser formulado con mayor precisión de la siguiente manera: demostrar la consistencia de un sistema formal $F$ consiste en demostrar por métodos finitarios que ninguna secuencia de fórmulas del tipo que constituye una demostración tiene como fórmula final una contradicción.

La teoría en la cual se realizan las investigaciones lógico-combinatorias de Hilbert a las cuales se refiere von Neumann es la llamada Teoría de la demostración. La expresión 'Teoría de la demostración', en alemán, Beweistheorie, fue introducida por Hilbert en 1922 para designar una nueva empresa científica cuya significación conceptual es la siguiente: "Como el físico investiga sus aparatos, el astrónomo su posición y el filósofo ejerce la crítica de la razón, así, en mi opinión, el matemático solamente asegura sus proposiciones a través de una crítica de la demostración y por esto él necesita una Teoría de la demostración." ${ }^{2}$ Sin duda alguna, no era poco ambiciosa la expectativa que Hilbert tenía en relación con su Teoría de la demostración. Más allá, es claro, del problema mismo de la consistencia de la matemática, el horizonte de cuestiones y temas que tal teoría se proponía tratar y cuya solución buscaba ya había sido enunciado en 1917: "el problema de la resolubilidad en principio de toda cuestión matemática, el problema de la verificabilidad (controlabilidad) posterior del resultado de una investigación matemática, aún más, la cuestión del criterio de simplicidad para las demostraciones matemáticas, de la relación entre contenido y formalismo (lnhaltlichkeit und Formalismus) en la matemática y la lógica y, finalmente, el problema de la decidibilidad de una cuestión matemática por medio de una número finito de operaciones" (Hilbert 1917, pp. 412-413).

Sin embargo, puede no ser irrelevante recordar que de hecho había una pregunta filosóficaepistemológica que se pretendía reducir a la solución de un problema matemático. Para la aclaración de esa cuestión filosófica-epistemológica previa, la lectura del trabajo de von Neumann sirve de invaluable apoyo. En particular, von Neumann omite que la reducción de la matemática a un sistema formal, al 'juego de fórmulas' al que de manera crítica se refería Hermann Weyl, es completamente ajena a las ideas de Brouwer, aunque en última instancia Heyting o el propio Weyl concedan en formalizar los procedimientos constructivos, lo cual ya podría ser demasiada concesión. Tampoco el logicismo concebía el simbolismo como manipulación reglada de símbolos sin significado. Para reducir la cuestión filosófica-epistemológica a un problema matemático sería necesario justificar esa concepción meramente simbólica de la matemática. Al respecto de Hilbert y de su teoría de la demostración, escribe von Neumann:
Su idea fundamental es la siguiente: aun si las afirmaciones con contenido de la matemática clásica resultaran no ser confiables, es cierto sin embargo que ésta involucra un procedimiento contenido en sí mismo, que procede de acuerdo con reglas seguras conocidas por todos los matemáticos, y que consiste en construir sucesivamente ciertas combinaciones de símbolos primitivos que pueden ser calificadas como "correctas" o "demostradas". (von Neumann 1931, pp. 116-117)

La idea de que la matemática involucra un procedimiento (simbólico) de acuerdo con reglas es, sin duda, una idea hilbertiana, pero aquello que llama Hilbert la 'idea fundamental' de su teoría de la demostración, que se encuentra reproducida en varios de sus textos, es su precondición:

\footnotetext{
2 Hilbert (1922), p. 170. Con alguna variación, pero sin utilizar la expresión ‘Teoría de la demostración', esta frase se encuentra en Hilbert (1918), p. 415

${ }^{3}$ En este volumen, p 79.
} 
El pensamiento fundamental de mi teoría de la demostración es el siguiente: todo lo que ha contado hasta este momento como matemática es susceptible de formalización rigurosa. De ese modo la propia matemática, es decir, la matemática en un sentido estricto, se convierte en un conjunto de fórmulas. (Hilbert 1931b, p. 489)

La reducción de la matemática a sistemas formales depende entonces de la tesis de formalización completa. Pero esta tesis no concluye en una tesis filosófica substantiva acerca de la naturaleza de la matemática en general, sino que es una tesis metodológica: la matemática es vista exclusivamente como procedimiento (que puede ser representado simbólicamente), pues esa es la manera de reducir el problema filosófico-epistemológico al problema matemático de la demostración de consistencia. Y por más que los procedimientos contemplados no sean constructivamente aceptables desde el punto de vista del contenido, lo cual los hace 'no confiables', el procedimiento de construcción de acuerdo con la noción formal de demostración que lo representa sí es confiable.

Ilustra von Neumann la crítica intuicionista considerando la demostración de una proposición que afirme la existencia de un número real x con una propiedad E, pero que de la demostración no pueda derivarse un procedimiento para construir tal x. Sin entrar en detalles de la demostración, algunas observaciones son pertinentes. En primer lugar, está involucrada la llamada 'Conjetura de Goldbach', a saber, que cualquier número par mayor que 2 es la suma de dos primos. Esa conjetura se expresa fácilmente en el lenguaje formal de la aritmética de Peano, pero la demostración considerada se desarrolla en la teoría analítica de números. El ejemplo elegido muestra indirectamente que la disputa con los intuicionistas en relación con la teoría usual de números era teórica más que práctica, pues las divergencias matemáticas efectivas, relacionadas con el principio de tercero excluido o con demostraciones no-constructivas de existencia se daban en el ámbito del análisis. Ahora bien, lo que se demuestra es, en verdad, una condicional: si la conjetura de Goldbach es falsa, una cierta propiedad vale para los impares, pero no para los pares, o la inversa, si vale para los pares, no vale para los impares. En cualquiera de los dos casos, no había todavía - no hay hoy tampoco- herramientas matemáticas disponibles para demostrar ninguno de ellos.

Dos observaciones sobre la prueba. En primer lugar, no es una demostración formal: ella tiene la forma usual de una prueba en la práctica matemática (para una audiencia competente en el tópico). Pero hay que recordar que está en operación el 'principio fundamental' de acuerdo con el cual toda prueba es formalizable. En segundo lugar, la prueba utiliza el principio de tercero excluido. Para Hilbert, el principio de tercero excluido es un principio lógico transfinito, por lo tanto, cuyo contenido es no confiable, pero no sin contenido, como lo prueba el siguiente pasaje:

Entre los axiomas y proposiciones de la lógica, Tertium non datur ocupa una posición distinguida: mientras que todos los demás axiomas y proposiciones se dejan de manera inmediata reducir sin dificultad a definiciones, Tertium non datur expresa un nuevo hecho contentualmente significativo que necesita de prueba. (Hilbert 1931a, pp. 124-125)

Tercero excluido es uno de esos procedimientos que todos los matemáticos conocen a los cuales se refería von Neumann, pero cuya utilización debe ser demostrada confiable por vía de una demostración de consistencia. Ahora bien, al formalizar la matemática, si hubiera un error en la derivación, se lo podría encontrar por un procedimiento finitario. Como escribe von Neumann:

En otra palabras, aunque no siempre es posible controlar finitariamente (esto es, en general) la aseveración de un teorema matemático clásico, sí es posible controlar el camino formal a través del cual se llegó a dicha aseveración. (von Neumann 1931, p. 117)

Así, de acuerdo con von Neumann, la confiabilidad de la matemática clásica debe consistir en una investigación de los métodos de demostración, y no de enunciados aislados:

\footnotetext{
${ }^{4}$ En este volumen, p. 79.
} 
Debemos concebir la matemática clásica como un juego combinatorio con los símbolos primitivos y establecer, de un modo combinatorio-finitario, a qué combinación de símbolos primitivos o “demostraciones" pueden conducir estos métodos de construcción. (von Neumann 1931, p. 117)

Pero ver la matemática como un juego combinatorio, que la debamos concebir así desde la perspectiva de los fundamentos, no es lo mismo que decir que la matemática es un juego combinatorio de manipulación simbólica de fórmulas desprovistas de significado. La matemática es un juego de esa naturaleza desde la perspectiva de la metamatemática o teoría de la demostración, pero inclusive esa reducción no es arbitraria: dejando de lado su valor matemático, ese juego de fórmulas, dice Hilbert, expresa la técnica de nuestro pensamiento, que es eminentemente simbólico. En efecto: En el principio era el signo.

\section{La teoría de la demostración}

En la segunda sección, von Neumann enumera las tareas que debe realizar la teoría de la demostración de Hilbert. Las tres primeras tienen que ver con la formalización de la lógica y de la matemática, que esencialmente ya fue llevada a cabo por Russell; la cuarta es la difícil: la demostración de consistencia. Advierte von Neumann que la formalización de la lógica y de la matemática puede ser llevada a cabo de varias maneras diferentes. De hecho, en "Sobre la teoría hilbertiana de la demostración", de 1927, von Neumann presenta un formalismo estilo Hilbert para un fragmento de la aritmética. En efecto, von Neumann hace uso de la típica presentación de Hilbert de sistemas axiomáticos en la cual cada grupo de axiomas busca caracterizar los diferentes conceptos involucrados, inclusive, pero en este caso, fundamentalmente, también los lógicos. Revisemos, recurriendo en algún momento también al trabajo de 1927, como se realizan las tres primeras tareas que enuncia von Neumann en 1931.

1. La primera es enumerar los símbolos usados en lógica y matemática. En "Sobre la teoría hilbertiana de la demostración", von Neumann distingue 5 clases de símbolos: la de variables (tanto proposicionales como de individuos); la de constantes (como ' $\mathrm{O}$ '); la de operaciones (que incluyen ' $\neg$ ' y '.', pero también de suma '+' y producto ' $x$ '); la de los símbolos de igualdad ' $=$ ' y de abstracciones (como los cuantificadores existencial y universal, ' $\Sigma$ ' y ' $\Pi$ ', respectivamente); y la de puntuación (como comas, etc.).

2. La segunda tarea consiste en caracterizar inequívocamente la clase de las fórmulas 'con sentido' de la matemática clásica, básicamente por medio de una definición recursiva. Esa clase, claro está, incluye no solamente las fórmulas verdaderas, sino también las falsas. En el trabajo de 1927, una regla de substitución juega un papel fundamental, pues estará involucrada en la formulación de los axiomas.

3. La última de esas tres tareas que se vinculan con la formalización de la lógica y la matemática clásicas consiste en presentar un procedimiento de construcción para las fórmulas demostrables que corresponden a las proposiciones de la matemática clásica. Dicho de otra manera, para el caso que nos ocupa, se trata de seleccionar un conjunto de fórmulas como axiomas y ofrecer una definición de demostración como secuencia de fórmulas, i.e., la definición de demostración formal que representa dentro del formalismo los resultados obtenidos en la práctica matemática clásica (algunos de cuyos principios y métodos de demostración son no-confiables).

La cuarta y última tarea es la demostración de consistencia, que von Neumann enuncia así en su trabajo de 1931:

4. Se debe mostrar (de un modo finitario-combinatorio) que aquellas fórmulas que corresponden a afirmaciones de la matemática clásica controlables desde un punto de vista finito (comprobables aritméticamente) podrán ser demostradas (i.e., construidas),

${ }^{5}$ En este volumen, pp. 79-80. 
según el procedimiento explicado en 3, sí y sólo sí el "procedimiento" efectivo recién mencionado de las afirmaciones matemáticas correspondientes da como resultado que son correctas. (von Neumann 1931, pp. 118-119)

La idea es establecer la confiabilidad de la matemática clásica como un atajo para justificar enunciados aritméticos cuya verificación por medios confiables seria tediosa. Con esto -parece decir von Neumann- sería suficiente para justificar la confiabilidad de la práctica matemática, confiabilidad que es vista como un hecho empírico. Ahora bien, si el check de una fórmula numérica muestra que esa fórmula es falsa, entonces de ella puede derivarse ' $p=q$ ' para dos numerales distintos. Dado (3), entonces se podría dar una demostración formal de ' $\mathrm{p}=\mathrm{q}$ ', de la cual se seguiría ' $1=2$ '. Luego, basta para demostrar la consistencia demostrar que no hay una demostración formal de ' $1=2$ '.

Así las cosas, el objetivo principal de Hilbert era un resultado de 'conservatividad', coherente con el tipo de interpretación que Prawitz denomina 'formalista moderada' (Prawitz 1981, p. 253). Tal resultado presupone, es claro, la distinción entre sentencias reales e ideales, que en este contexto metamatemático son aquellas que involucran la referencia a una totalidad infinita de objetos concebidas como 'redondeando' la teoría, esto es, permitiendo obtener resultados de manera 'más fácil' sobre la parte real, concebida de acuerdo con el mismo contexto como verificables. Si uno piensa que esas sentencias ideales introducen 'elementos ideales', entonces el problema sería mostrar que el uso de esos elementos ideales en demostraciones de enunciados reales en el sentido de verificables no lleva a resultados incorrectos. Ahora bien, ¿bajo qué condiciones ese resultado de conservatividad es equivalente al problema de la consistencia?

De acuerdo con Prawitz (1981, p. 257), la formulación del proyecto, dada la formalización de una teoría matemática $\mathrm{T}$ adquiere la siguiente forma:

Para toda demostración $\mathrm{P}$ en $\mathrm{T}$ y para toda sentencia real $\mathrm{A}$ en $\mathrm{T}$, si $\mathrm{P}$ es una demostración de $\mathrm{A}$ en $\mathrm{T}$, entonces $\mathrm{A}$ es verdadera.

Este condicional es solamente uno de los lados del formulado por von Neumann. La estructura de la prueba de ese condicional sería la siguiente:

1. En primer lugar, se prueba la contrapositiva.

2. Supóngase una sentencia numérica $E$ que por un procedimiento finitario es mostrada como falsa/incorrecta.

3. Se puede entonces derivar de esa fórmula la relación $\mathrm{p}=\mathrm{q}$ para dos números efectivamente dados.

4. Dado (3) se puede producir una demostración formal de $p=q$.

5. De esa demostración, se puede trivialmente producir una demostración formal de $1=2$.

6. El formalismo no demuestra $1=2$.

7. Luego, si el enunciado (verificable por métodos finitarios) que corresponde a una fórmula A del formalismo es verificado falso, entonces A no puede ser demostrada en el formalismo.

8. Luego, por contrapositiva, dada una fórmula A que corresponde a un enunciado de la matemática clásica que puede ser 'checada' (verificada) por medios finitarios, si ella puede ser demostrada en el formalismo, entonces el enunciado que corresponde a $\mathrm{A}$ es verificado como verdadero.

\section{Los resultados parciales}

La manera en que (3) es llevada a cabo por von Neumann no es explicitada en su artículo, pero destaca sus dos elementos fundamentales que se relacionan con la noción de demostración:

\footnotetext{
${ }^{6}$ En este volumen, p. 80
} 
$3_{1}$. En primer lugar, caracterizar de manera unívoca y finitaria algunas fórmulas que son llamadas 'axiomas'.

32. En segundo lugar, caracterizar la noción de fórmula demostrada.

En el artículo de 1927, hay seis grupos de axiomas. Los axiomas llamados finitos son: axiomas para la lógica proposicional (formulados exclusivamente con ' ' y' 'ר'); los axiomas para igualdad '=’; los axiomas para números no negativos ( 0 es un número, si a es un número, el sucesor también lo es; 0 no es sucesor de ningún número, si sucesores de a y b son iguales, a y b también son iguales). Los axiomas para cuantificadores son transfinitos: ellos implican, en particular, el principio de tercero excluido. Hay dos grupos de axiomas más, para funciones y para definiciones, que son también transfinitos. El lector habrá notado que el sistema de 1927 no contiene explícitamente el principio de inducción, aunque los axiomas dados sí contienen una versión más débil del mismo. Se trata, por lo tanto, de un fragmento de la aritmética clásica, que podría ser completado.

Los axiomas se consideran demostrados. Además, si $a$ y $b$ son dos fórmulas, y a y $a \rightarrow b$ han sido demostradas, entonces $b$ también ha sido demostrada. La demostración es una secuencia checable de fórmulas. Así queda completa la manera de Hilbert de ver la matemática como un stock de fórmulas o un juego combinatorio: en ella las fórmulas difieren de las fórmulas usuales de la matemática en la medida en que incluyen los símbolos lógicos; además, una demostración es una figura intuitivamente presentada que consiste en inferencias donde cada premisa es un axioma o es la fórmula final de una inferencia a partir de fórmulas anteriores en la secuencia o resulta de una fórmula por substitución. En relación con la inferencia en el sentido usual de la matemática escribe:

En lugar de la inferencia con contenido, en la teoría de la demostración cuenta una acción externa de acuerdo con reglas, es decir, el uso de esquemas de inferencia y de substitución. (Hilbert 1931b, pp. 489 . 490)

Una fórmula es formalmente demostrable si es un axioma o la fórmula final de una demostración, pero la metamatemática o teoría de la demostración, donde se prueba la consistencia de la teoría de que se trate, es matemática con contenido en un sentido muy específico. En efecto, en la metamatemática solamente pueden utilizarse conceptos y métodos con contenido finitario (o intuicionísticamente aceptables, como quiere von Neumann) para demostrar la consistencia de la teoría axiomática formal de que se trate. En el artículo que acabamos de citar, Hilbert menciona las demostraciones de Ackermann y von Neumann sobre la consistencia de fragmentos más débiles de la aritmética clásica. En particular, sobre el fragmento que caracterizamos muy esquemáticamente antes, von Neumann había obtenido la correspondiente demonstración de consistencia. Al final de su trabajo de 1931, von Neumann declara con entusiasmo que aunque todavía no se ha demostrado la consistencia de la matemática clásica, se ha demostrado la consistencia de un sistema más débil que la matemática clásica (el de 1927). Y agrega:

De este modo, el sistema de Hilbert ha superado la primera prueba de fuerza: la justificación de un sistema matemático no finitario y no puramente constructivo es alcanzada a través de medios finitariosconstructivos. Si se conseguirá extender esta justificación para sistemas más complicados e importantes de la matemática clásica, sólo el futuro lo dirá. (von Neumann 1931, p. 121)7

En la tragedia griega perfecta el reconocimiento coincide con el cambio de fortuna: en el mismo seminario, Kurt Gödel presentará el primero de sus resultados acerca de la incompletitud de la aritmética. De inmediato, von Neumann entrevió una consecuencia del teorema que Gödel enunciaría poco después: la consistencia de la aritmética, bajo pena de inconsistencia, no podría demostrarse con métodos que pudieran representarse dentro de ella, como se supone que deberían ser los finitarios o constructivos. La reacción algo desengañada de von Neumann fue abandonar los estudios fundacionales para concentrarse en cuestiones relativas a la aplicación de la matemática. Para él, la

\footnotetext{
${ }^{7}$ En este volumen, p. 82.
} 
cuestión filosófica-epistemológica de la confiabilidad de la matemática clásica ya no podría ser resuelta por el camino indicado por Hilbert. ${ }^{8}$

La reacción de Hilbert no fue la de von Neumann, que fue mucho más filosófica de lo que acaso él mismo pudiera aceptar. La reacción fue la de matemáticos que enfrentan en la solución de un problema dificultades que no por inesperadas dejan de ser inusuales en la historia de la matemática, a saber, que los medios considerados suficientes para la resolución de un problema no son tales. Luego, deben utilizarse métodos más poderosos, pero en este caso en especial, con restricciones filosóficoepistemológicas bien justificadas. Como dirá Paul Bernays, debe extenderse el cuadro de los métodos finitariamente aceptables. En este respecto, las contribuciones de Gerhard Gentzen fueron, sin lugar a dudas, las fundamentales.

Entre 1934 y 1939 Gentzen produjo cuatro diferentes demostraciones de consistencia de la aritmética clásica (PA). La primera prueba, de 1934, no fue publicada. Después de enviarla a Gödel y a von Neumann, Gentzen desiste de su publicación en razón de las críticas recibidas: la demostración no sería constructiva/finitaria. En 1968, en un congreso sobre teoría de la demostración e intuicionismo, Bernays presenta una ponencia, publicada posteriormente en 1970, en la cual recuerda esas críticas sobre la primera prueba de Gentzen. En ese artículo escribe:

La primera prueba de consistencia del sistema formal de la teoría de primer orden de números publicada por Gentzen, incluyendo la lógica estándar, los axiomas de Peano y las definiciones recursivas, fue dada en el artículo de Gentzen 'Die Wiederspruchsfreiheit der reinen Zahlentheorie' (Math. Ann. 112 (1936)). Sin embargo, esa no fue la prueba original, sino una versión revisada de ella. La revisión fue motivada por una crítica a la prueba original, con la cual en aquel entonces yo también estuve de acuerdo, cuyo fundamento era que ella incluía una aplicación del Teorema del abanico. Gentzen no se opuso explícitamente a esa opinión; él cuidó de la crítica modificando su prueba de consistencia antes de que fuera publicada. Afortunadamente, el texto de la prueba original fue preservado en las pruebas de galera. (Bernays 1970, p. 409)

Fue solamente en la segunda prueba, de 1936, que fue la primera publicada, y en la tercera, de 1938, que aparece el uso de inducción transfinita hasta $\varepsilon_{0}$. La cuarta demostración, presentada en 1942 como Tesis de Habilitación y publicada en 1943, se basa de hecho en un resultado de 'indemostrabilidad'. En efecto, Gentzen formaliza la inducción transfinita hasta $\varepsilon_{0}$ en una sentencia del lenguaje de PA y prueba que esa sentencia no es demostrable en PA, garantizando así que al menos una sentencia no es demostrable en PA, i.e., que PA es consistente. (Gentzen también probó que todos los casos de inducción transfinita hasta ordinales menores que $\varepsilon_{0}$ son demostrables en PA.) Ahora bien, de hecho, Gentzen había producido antes una quinta demostración, iqué es históricamente la primera! En 1933 Gentzen había publicado una interpretación de la aritmética clásica en la aritmética intuicionista de Heyting y muestra que, como resultado de esa interpretación, si la aritmética intuicionista es consistente, entonces la aritmética clásica también lo es. ¡Ese resultado es explícitamente una reducción del problema de la consistencia de la aritmética clásica al problema de la aritmética intuicionista! Se trata del mismo espíritu 'reduccionista' que preside los resultados de traducción/interpretación /codificación de final de los años veinte e inicio de los treinta del siglo pasado.

Una observación final de método es aquí pertinente. von Neumann tiene razón cuando afirmaba, en 1931, que la cuestión de la confiabilidad de la matemática clásica se había transformado, "en las últimas décadas", en un problema lógico-matemático. De hecho, dicho de manera acaso algo grosera, los dos principales (y tradicionales) programas de fundamentación de la matemática, a saber, el logicismo y el formalismo, dependían para su consecución de resultados matemáticos. Ahora bien, no es en general claro como una investigación filosófica puede depender de un resultado matemático (o también, si vamos al caso, de un resultado empírico). Wittgenstein tal vez diría que si una cuestión filosófica pudiera ser resuelta por una prueba matemática (o, si vamos también al caso, por un experimento), eso mostraría que la cuestión no era una cuestión filosófica. Conocemos la historia: sin

\footnotetext{
${ }^{8}$ Ver Murawski (2004), secs. 4 y 5.
} 
considerar los 'new' tan caros para ciertas posturas revisionistas, esos programas naufragaron, al menos en sus formulaciones originales, por fuerza de resultados matemáticos.

No obstante, al naufragio algo sobrevivió: sobre el suelo firme de los resultados de Gentzen la teoría de la demostración se consolidó como una de las cuatro grandes áreas de la lógica, tanto en su versión reductiva, ciertamente más próxima del proyecto hilbertiano, cuanto en su versión general, libre de cualquier expectativa fundacional, que busca investigar "pruebas", sin imponer restricciones a los métodos utilizados en la investigación. ${ }^{9}$ La teoría de la demostración produjo y produce frutos técnicos de inequívoca importancia e, inclusive, se muestra como una perspectiva interesante semántica de naturaleza constructiva, un hecho que acaso sería sorprendente para von Neumann así como para el formalismo de manera general.

Bibliografía

Bernays, P. (1970), “On the Original Gentzen Consistency Proof for Number Theory”, en Kino, A., Myhill, J. y R. E. Wesley, (eds.), Intuitionism and Proof Theory: Proceedings of the Summer Conference at Buffalo N.Y., Amsterdam: NorthHolland, pp. 409-417.

Gentzen, G. (1936), "Die Widerspruchsfreiheit der reinen Zahlentheorie”, Mathematische Annalen 112: 493-565.

Gentzen, G. (1938), “Neue Fassung des Wiederspruchfreiheis-beweises für die reine Zahlentheorie”, Forschungen zur Logik und zur Grundlegung der exakten Wissenschaften 4: 19-44.

Gentzen, G. (1969), The Collected Papers of Gerhard Gentzen (ed. por M. Szabo), Amsterdam: North-Holland.

Hilbert, D. (1918), “Axiomatisches Denken”, Mathematische Annalen 78: 405-415.

Hilbert D. (1922), "Neubegründung der Mathematik. Erste Mitteilung", Abhandlungen aus dem mathematischen Seminar der Hamburgischen Universität I: 157-177. (Reimpreso en: Hilbert, D., Gesammelte Abhandlungen, Vol. III, Berlin: Springer, 1935, pp. 157-177.)

Hilbert, D. (1931a), "Beweis des Tertium non datur", Nachrichten von der Gesellschaft der Wissenschaften zu Göttingen, Mathematisch-physikalische Klasse (1930): 120-125.

Hilbert, D. (1931b), "Die Grundlegung der elementaren Zahlenlehre", Mathematische Annalen 104: 485-494.

Murawsky, J. (2004), "John von Neumann and Hilbert's School of Foundations of Mathematics", Studies in Logic, Grammar and Rhetoric 7: 37-55.

Prawitz, D. (1981), "Philosophical Aspects of Proof Theory", en Fløistad, G. (ed.), Contemporary Philosophy: A New Survey, Volume 1, Philosophy of Language, Philosophical Logic, Dordrecht/Boston/Lancaster: Martinus Nijhoff Publishers, pp. 235-277.

von Neumann, J. (1927), "Zur Hilbertschen Beweistheorie”, Mathematische Zeitschrift 26: 1-46.

von Neumann, J. (1931), "Die formalistische Grundlegung der Mathematik", Erkenntniss 2: 116-121. (Seguimos la excelente traducción de Eduardo Giovaninni, este volumen, pp. 79-82.)

\footnotetext{
9 La distinción entre una "teoría general de la demostración" y una "teoría reductiva de la demostración" fue introducida en Prawitz (1971).
} 